\title{
Distributed Learning Algorithms and Lossless Convex Relaxation for Economic Dispatch with Transmission Losses and Capacity Limits
}

\author{
Kwang-Ki K. Kim (iD) \\ Department of Electrical Engineering, Inha University, Incheon 22212, Republic of Korea \\ Correspondence should be addressed to Kwang-Ki K. Kim; kwangki.kim@inha.ac.kr
}

Received 14 March 2019; Accepted 27 May 2019; Published 13 June 2019

Guest Editor: Michael Z. Q. Chen

Copyright (C) 2019 Kwang-Ki K. Kim. This is an open access article distributed under the Creative Commons Attribution License, which permits unrestricted use, distribution, and reproduction in any medium, provided the original work is properly cited.

\begin{abstract}
This paper considers problems of economic dispatch in power networks that contain independent power generation units and loads. For efficient distributed economic dispatch, we present a mechanism of multiagent learning in which each agent corresponding to a generation unit updates the power generation based on the received information from the neighborhood. The convergence of the proposed distributed learning algorithm to the global optimal solution is analyzed. Another method of distributed economic dispatch we propose is a decentralized iterative linear projection method in which the necessary optimality conditions are solved without considering the generation capacities and the obtained solutions are iteratively projected onto the convex set corresponding to the generation capacities. A centralized method based on semidefinite programming for economic dispatch with a loss coefficient matrix is also presented for comparisons. For demonstration, the proposed methods of distributed economic dispatch are applied to a 6-generator test case and the three different methods of economic dispatch give the same solutions. We also analyze parametric dependence of the optimal power generation profiles on varying power demands in economic dispatch.
\end{abstract}

\section{Introduction}

The smart grid infrastructure including smart sensors and meters and communication technology has triggered revisiting fundamental problems of power systems research. In such energy system infrastructures with communication networks, different independent units take the control and operational responsibility in different areas of the system $[1,2]$.

In particular, economic dispatch with integration of distributed generators and energy storage systems in a smart grid infrastructure is one of the major challenges for largescale complex power networks to meet the total power demand by allocating demand among many independently operated distributed generators in an efficient way with guaranteed quality of service and safety [3]. Because of distributed and hierarchical structures of power supplies and demands, and their connections through information and communication infrastructures, it requires distributed mechanisms of dispatching electrical power demands among available generation units in efficient ways. Several distributed algorithms for economic dispatch have been presented in the literature, whereas many of existing approaches to economic dispatch are centrally performed. In $[4,5]$, the authors propose the distributed incremental cost consensus algorithm in which the dual variable corresponding to the marginal price and the power generation mismatches are updated by a weightedsum consensus rule. But such algorithms do not consider any transmission constraints.

Other consensus-based distributed algorithms for economic dispatch are presented by different research groups. For the method presented in [6], each generator learns the mismatch between demand and total power generation by communication over a strongly connected graph and corrects its own power generation to achieve the consensus. In $[7,8]$, the authors present a consensus-based distributed bisection method in which the dual variable corresponding to the marginal price is explicitly computed by a bisection method with power generation updates of distributed generators. In addition, the authors of [9] propose a distributed method of lambda-iteration in which the conventional lambda-iteration method [10] is modified to take the presence of prohibited 
operating zones and to avoid an oscillatory phenomenon. In [11], a game-theoretic learning distributed algorithm based on population dynamics without considering transmission losses is presented. Another game-theoretic approach is presented by the authors of [12] in which a cooperative game is formulated for optimal power aggregation to minimize the total generation cost while meeting the generation capacity constraints. Evolutionary algorithms are also applied to the problem of economic dispatch: genetic algorithm [13], particle swarm optimization [14], and evolutionary programming techniques [15].

Our contribution is threefold. First, we present a method of primal-dual iteration for computing optimal power generation profiles in economic dispatch with transmission losses and generation limits. The proposed algorithm is based on distributed computations of the independent decisionmaking processes with information exchange. The convergence of the proposed algorithm to the global optimum is analyzed. Secondly, semidefinite programming relaxation of the associated quadratic constrained quadratic program formulated from a constrained economic dispatch problem. This convex relaxation is shown to be exact; i.e., strong duality holds. Thirdly, we present a decentralized method of iterative linear projection for the same class of economic dispatch. This algorithm is based on iterative projections of linear system solution corresponding to the reduced KKT conditions and its convergence is guaranteed with relatively small size of iteration numbers. In addition, the explicit dependence of the optimal power generation profiles on varying power demand is investigated in terms of multiparametric programming. This paper is organized as follows.

Section 2 presents a distributed iteration method for economic dispatch in the presence of generation capacity limits and quadratic transmission losses. In Section 3, a semidefinite programming relaxation of the associated quadratic constrained quadratic program is studied and its strong duality is analyzed. In addition, the explicit parametric dependence of the optimal generation profiles on varying power demand is investigated. To demonstrate the effectiveness of the proposed distributed and convex relaxation algorithms, the methods are applied to the IEEE 26-bus 6-generator test case in Section 4. Section 5 concludes this paper.

\section{Distributed Economic Dispatch in Smart Grids}

2.1. A Brief Introduction to Economic Dispatch. Economic dispatch is an optimization problem in which the objective is to minimize the total power generation cost under physical limitations of distributed generators and demandsupply balance that are represented as inequality and equality constraints:

$$
\text { ED } \begin{cases}\text { minimize } & \sum_{i=1}^{N} f_{i}\left(p_{i}\right) \\ \text { subject to } \quad & p_{i}^{\min } \leq p_{i} \leq p_{i}^{\max } ; i=1,2, \ldots, N \\ & \sum_{i=1}^{N} p_{i}-\sum_{i=1}^{N} \sum_{j=1}^{N} p_{i} B_{i j} p_{j}=P_{d}\end{cases}
$$

where $p_{i}$ refers to the power generation output of the generator $i$, and $p_{i}^{\min }$ and $p_{i}^{\max }$ are the minimum and maximum generation output limits of the generator $i$, respectively. The power loss in transmission is given as a quadratic form parameterized by the loss-coefficient matrix $B$ [10]. The power generation cost functions $f_{i}: \mathbb{R}_{+} \longrightarrow \mathbb{R}_{+}$for $i=$ $1,2, \ldots, N$ are usually modeled as quadratic forms:

$$
f_{i}\left(p_{i}\right)=\frac{1}{2} a_{i} p_{i}^{2}+b_{i} p_{i}+c_{i}
$$

where $a_{i}\left(\left[\$ / \mathrm{MW}^{2}\right]\right), b_{i}([\$ / \mathrm{MW}])$, and $c_{i}([\$])$ denote the cost coefficients of generator $i$.

2.2. Optimality Conditions for Economic Dispatch. Consider a Lagrange function (or Lagrangian) defined by

$$
\begin{aligned}
& L\left(p_{1}, p_{2}, \ldots, p_{N}, \mu\right) \\
& \quad:=\sum_{i=1}^{N} f_{i}\left(p_{i}\right)+\mu\left(p_{d}+\sum_{i=1}^{N} \sum_{j=1}^{N} p_{i} B_{i j} p_{j}-\sum_{i=1}^{N} p_{i}\right)
\end{aligned}
$$

for which the equality constraint corresponding to the power balance is relaxed and integrated into the objective function by introducing a Lagrange multiplier $\mu \in \mathbb{R}$. With this definition of Lagrangian, the necessary conditions for optimality are given as follows:

(1) Stationarity:

$$
\nabla_{p_{i}} L\left(p_{1}^{*}, p_{2}^{*}, \ldots, p_{N}^{*}, \mu^{*}\right)=0 \text { for } i=1,2, \ldots, N
$$

(2) Primal feasibility:

$$
\begin{aligned}
P_{d}+\sum_{i=1}^{N} \sum_{j=1}^{N} p_{i}^{*} B_{i j} p_{j}^{*}-\sum_{i=1}^{N} p_{i}^{*} & =0 \\
p_{i}^{\min } \leq p_{i}^{*} \leq p_{i}^{\max } & \text { for } i=1,2, \ldots, N
\end{aligned}
$$

To find an optimal demand allocation in economic dispatch (1), we need to solve $N+1$ equations while satisfying $2 N$ inequalities for $N+1$ variables $\left(p_{1}^{*}, p_{2}^{*}, \ldots, p_{N}^{*}, \mu^{*}\right)$ in $(4) \sim(6)$.

2.3. Multiagent Learning for Distributed Economic Dispatch. To find a solution profile $\left(p_{1}^{*}, p_{2}^{*}, \ldots, p_{N}^{*}, \mu^{*}\right)$ that satisfies the optimality conditions (4) (6), we develop an iterative method of distributed optimization that is based on multiagent learning. Figure 1 shows a schematic diagram for message exchanges and updates among the aggregator (denoted by Node 0 ) and the agents (indexed by $1,2, \ldots, N$ ). Best response dynamics of power generation updates for agents and gradient descent dynamics of price updates for the aggregator are depicted in Figure 2 as block diagrams with arrows denoting message exchanges.

2.3.1. Primal Updates (Distributed Learning). Define sets $\mathcal{N}_{i} \triangleq\left\{j: B_{i j} \neq 0\right\}$ for $i=1,2, \ldots, N$ and the associated vector 


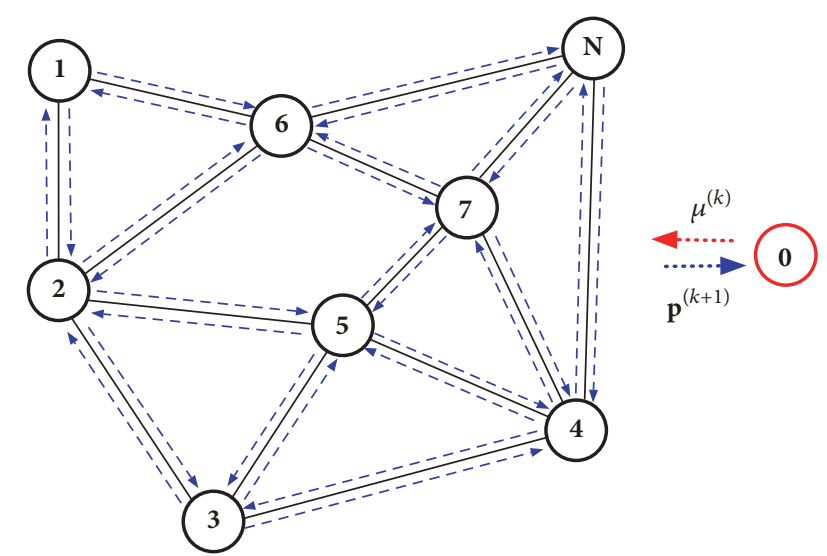

FIgURE 1: A schematic diagram for physical connections and communication in power-generation grid. The solid black lines denote the electrical connections between generators, the blue dashed arrows denote communication links between generators, the red dotted arrow denotes the broadcasting of the Lagrange multiplier, and the blue dotted arrows denote the generators' willingto-supply powers for given Lagrange multiplier.

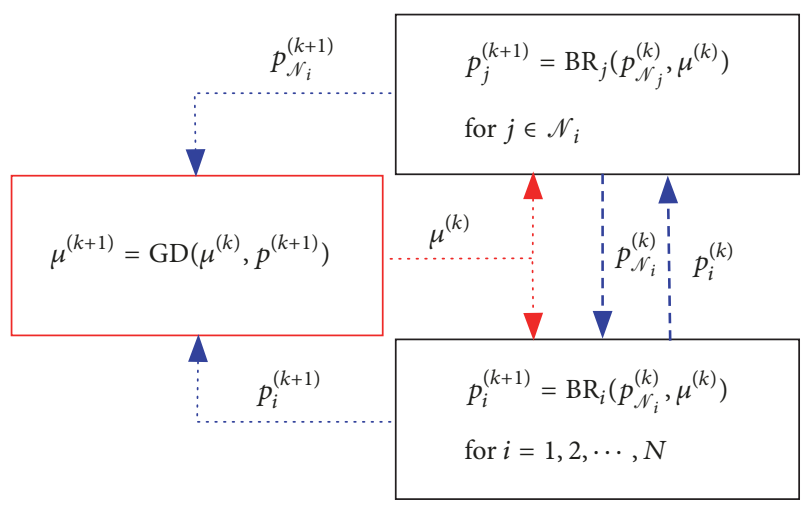

FIGURE 2: Block diagram of distributed iterative computations of primal-dual variables in economic dispatch with coupled power loss. The update rules $\mathrm{BR}_{i}$ and $\mathrm{GD}_{i}$ are defined in (10) and (11), respectively.

$p_{\mathcal{N}_{i}}=\left(p_{j}\right)_{j \in \mathcal{N}_{i}} \in \mathbb{R}^{\left|\mathcal{N}_{i}\right|}$, where $\left|\mathcal{N}_{i}\right|$ denotes the cardinality of the set $\mathcal{N}_{i}$. Each unit of the $i$ th generator receives the current computation of $p_{\mathcal{N}_{i}}$ from its neighbourhood $\mathcal{N}_{i}$ and the current computation of Lagrangian multiplier $\mu$ and updates its computation of $p_{i}$ by solving a local optimization

$$
p_{i}^{(k+1)}:=\arg \min _{p_{i}^{\min } \leq p_{i} \leq p_{i}^{\max }} L_{i}\left(p_{i}, p_{\mathcal{N}_{i}}^{(k)}, \mu^{(k)}\right)
$$

where

$$
\begin{aligned}
L_{i}\left(p_{i}, p_{\mathcal{N}_{i}}^{(k)}, \mu^{(k)}\right) \triangleq & f_{i}\left(p_{i}\right) \\
& +\mu^{(k)}\left(2 p_{i} \sum_{j \in \mathcal{N}_{i}} B_{i j} p_{j}^{(k)}-p_{i}\right)
\end{aligned}
$$

for $k=0,1, \ldots$ and for each $i=1,2, \ldots, N$. More succinctly, we write

$$
p_{i}^{(k+1)}:=\mathrm{BR}_{i}\left(p_{\mathcal{N}_{i}}^{(k)}, \mu^{(k)}\right) \quad \text { for } i=1,2, \ldots, N
$$

where the $i$ th local optimization (7) has a unique solution for every $k=0,1, \ldots$, provided that $a_{i} \neq 0$ due to its strict convexity. We note that the $i$ th local optimization (7) has the following closed-form solution:

$$
p_{i}^{(k+1)}= \begin{cases}p_{i}^{\min } & \text { for } \tilde{p}_{i}^{(k)}<p_{i}^{\min } \\ p_{i}^{\max } & \text { for } \tilde{p}_{i}^{(k)}>p_{i}^{\max } \\ \tilde{p}_{i}^{(k)} & \text { for } p_{i}^{\min } \leq \tilde{p}_{i}^{(k)} \leq p_{i}^{\max }\end{cases}
$$

where $\tilde{p}_{i}^{(k)} \triangleq\left(1 / a_{i}\right)\left(\mu^{(k)}\left(1-\sum_{j \in \mathcal{N}_{i}} B_{i j} p_{j}^{(k)}\right)-b_{i}\right)$.

2.3.2. Dual Update. The Lagrangian multiplier is updated to enforce the demand-supply balance to be satisfied. The aggregator receives the current computations of $p_{i}$ for $i=$ $1, \ldots, N$ and revises its previous computation of $\mu$ by

$$
\mu^{(k+1)}:=\mu^{(k)}+\alpha^{(k+1)}\left(P_{d}+P_{L}^{(k+1)}-\sum_{i=1}^{N} p_{i}^{(k+1)}\right)
$$

where $P_{L}^{(k+1)} \triangleq \sum_{i=1}^{N} \sum_{j \in \mathcal{N}_{i}} p_{i}^{(k+1)} B_{i j} p_{j}^{(k+1)}$ denotes the power loss computed for iteration steps $k=0,1, \ldots$, and $\alpha^{(k+1)}>0$ is a user-defined step size.

Figure 2 shows how such primal and dual updates are performed with exchange of information among neighboring agents (or generators) and the aggregator.

2.4. Decentralized Relaxed Convex $Q P$. Consider a simpler case of ED in (1) for which the power loss coefficients $B_{i j}$ $(i, j=1,2, \ldots, N)$ are neglected:

$$
\mathrm{ED}^{\prime} \begin{cases}\text { minimize } & \sum_{i=1}^{N} f_{i}\left(p_{i}\right) \\ \text { subject to } & p_{i}^{\min } \leq p_{i} \leq p_{i}^{\max } ; i=1,2, \ldots, N \\ & \sum_{i=1}^{N} p_{i}=P_{d}\end{cases}
$$

For this case with quadratic cost functions in (2), necessary conditions for optimality are given as the following linear system:

$$
\left[\begin{array}{ccccc}
a_{1} & 0 & \cdots & 0 & -1 \\
0 & a_{2} & \ddots & \vdots & -1 \\
\vdots & \ddots & \ddots & 0 & \vdots \\
0 & \cdots & 0 & a_{N} & -1 \\
1 & 1 & \cdots & 1 & 0
\end{array}\right]\left[\begin{array}{c}
p_{1}^{*} \\
p_{2}^{*} \\
\vdots \\
p_{N}^{*} \\
\mu^{*}
\end{array}\right]=\left[\begin{array}{c}
-b_{1} \\
-b_{2} \\
\vdots \\
-b_{N} \\
P_{d}
\end{array}\right]
$$

and

$$
p_{i}^{\min } \leq p_{i} \leq p_{i}^{\max } \text { for } i=1,2, \ldots, N .
$$

Note that if $a_{i} \neq 0$ for all $i$ so that system (13) is linearly independent, then we have a unique closed-form solution for (13): 


$$
\begin{aligned}
& p_{i}^{*}=\frac{1}{a_{i}}\left(\delta \beta+\delta P_{d}-b_{i}\right) \quad \text { for } i=1,2, \ldots, N \\
& \mu^{*}=\delta\left(\beta+P_{d}\right)
\end{aligned}
$$

where $\delta=\left(\sum_{i=1}^{N} 1 / a_{i}\right)^{-1}$ and $\beta=\sum_{i=1}^{N} b_{i} / a_{i}$. The vector $\mathbf{p}=$ $\left[\begin{array}{llll}p_{1}^{*} & p_{2}^{*} & \cdots & p_{N}^{*}\end{array}\right]^{\top} \in \mathbb{R}^{N}$ obtained in the closed-form (15) does not necessarily satisfy the capacity constraints (14).

Define the following sets of indices:

$$
\begin{aligned}
& \mathscr{I}_{\text {min }}(\mathbf{p}) \triangleq\left\{i: p_{i}<p_{i}^{\min }\right\} \\
& \mathscr{I}_{\text {max }}(\mathbf{p}) \triangleq\left\{i: p_{i}>p_{i}^{\max }\right\}
\end{aligned}
$$

Let $\mathscr{I}(\mathbf{p}) \triangleq \mathscr{I}_{\min }(\mathbf{p}) \cup \mathscr{I}_{\max }(\mathbf{p})$ define its union and a complementary set $\widetilde{\mathscr{I}}(\mathbf{p}) \triangleq\{1,2 \ldots, N\}-\mathscr{I}_{\text {max }}(\mathbf{p})$. For the given solution vector $\mathbf{p}$ obtained in (15), consider the following reduced economic dispatch

$$
\mathrm{ED}^{\prime}(\mathbf{p}) \begin{cases}\operatorname{minimize} & \sum_{i \in \widetilde{\mathscr{I}}(\mathbf{p})} f_{i}\left(p_{i}\right) \\ \text { subject to } & p_{i}^{\min } \leq p_{i} \leq p_{i}^{\max } ; i \in \widetilde{\mathscr{I}}(\mathbf{p}) \\ & \sum_{i \in \widetilde{\mathcal{F}}(\mathbf{p})} p_{i}=P_{d}-\sum_{i \in \mathscr{\mathcal { F }}_{\max }(\mathbf{p})} p_{i}^{\max }\end{cases}
$$

where the generators corresponding to the set $\mathscr{I}_{\max }(\mathbf{p})$ are assigned to produce their upper limits in capacity and the remaining generators are further considered as design variables for optimization. Similar to (15), a closed-form solution satisfying necessary conditions for optimality is obtained as follows:

$$
\begin{aligned}
& p_{i}^{*}= \begin{cases}p_{i}^{\max } & \text { for } i \in \mathscr{I}_{\max }(\mathbf{p}) \\
\frac{1}{a_{i}}\left(\widetilde{\delta} \widetilde{\beta}+\widetilde{\delta} \widetilde{P}_{d}-b_{i}\right) & \text { for } i \in \widetilde{\mathscr{I}}(\mathbf{p})\end{cases} \\
& \mu^{*}=\widetilde{\delta}\left(\widetilde{\beta}+\widetilde{P}_{d}\right)
\end{aligned}
$$

where $\widetilde{\delta}=\left(\sum_{i \in \widetilde{F}} 1 / a_{i}\right)^{-1}, \widetilde{\beta}=\sum_{i \in \mathcal{F}} b_{i} / a_{i}$, and $\widetilde{P}_{d}=$ $P_{d}-\sum_{i \in \mathscr{I}_{\max }(\mathbf{p})} p_{i}^{\max }$. Note that this update again does not guarantee satisfaction of the capacity constraints and this process must be iteratively performed. This iteration method stops when the set of saturated generators are the same in subsequent computations, i.e., $\widetilde{\mathscr{I}}\left(\mathbf{p}^{(k)}\right) \equiv \widetilde{\mathscr{I}}\left(\mathbf{p}^{(k-1)}\right)$, and the resultant optimal solution is given by

$$
p_{i}^{*}= \begin{cases}p_{i}^{\min } & \text { for } i \in \mathscr{I}_{\min }\left(\mathbf{p}^{(k)}\right) \\ p_{i}^{\max } & \text { for } i \in \mathscr{I}_{\max }\left(\mathbf{p}^{(k)}\right) \\ \frac{1}{a_{i}}\left(\widetilde{\delta} \widetilde{\beta}+\widetilde{\delta} \widetilde{P}_{d}-b_{i}\right) & \text { for } i \in \widetilde{\mathscr{I}}\left(\mathbf{p}^{(k)}\right)\end{cases}
$$

where

$$
\begin{aligned}
\widetilde{\delta} & =\left(\sum_{i \in \widetilde{F}\left(\mathbf{p}^{(k)}\right)-\mathcal{F}_{\min }\left(\mathbf{p}^{(k)}\right)} \frac{1}{a_{i}}\right)^{-1}, \\
\widetilde{\beta} & =\sum_{i \in \widetilde{\mathcal{F}}\left(\mathbf{p}^{(k)}\right)-\mathcal{I}_{\min }\left(\mathbf{p}^{(k)}\right)} \frac{b_{i}}{a_{i}} \\
\widetilde{P}_{d} & =P_{d}-\sum_{i \in \mathscr{F}_{\max }\left(\mathbf{p}^{(k)}\right)} p_{i}^{\max }-\sum_{i \in \mathscr{F}_{\min }\left(\mathbf{p}^{(k)}\right)} p_{i}^{\min } .
\end{aligned}
$$

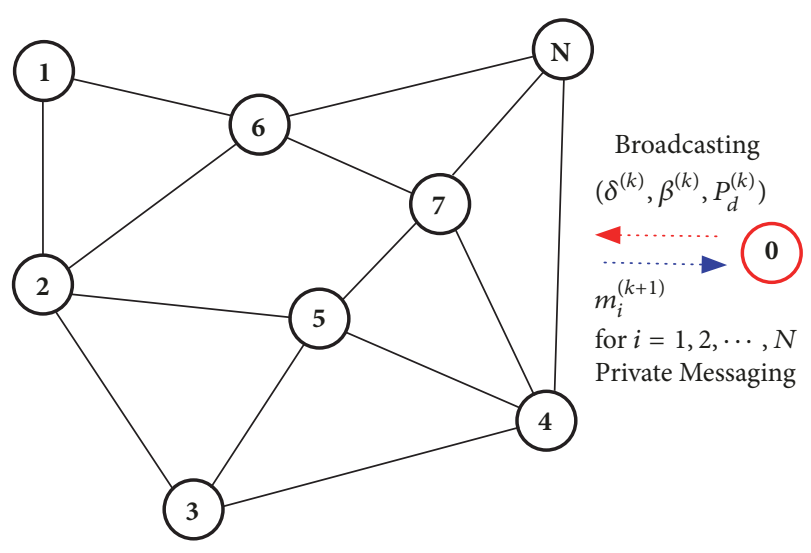

FIGURE 3: A schematic diagram for decentralized iterative computations and message passing. The aggregator (Node 0) broadcasts the values of $\left(\delta\left[\$ / \mathrm{MW}^{2}\right], \beta[\mathrm{MW}]\right)$ and $P_{d}[\mathrm{MW}]$, and each generator $i$ (Node $i$ ) sends a private message $m_{i}=$ $\left(a_{i}\left[\$ / \mathrm{MW}^{2}\right], b_{i}[\$ / \mathrm{MW}], s_{i} \in\{-1,0,1\}\right)$ to the aggregator, where $s_{i}=-1$ implies the $i$ th generator decides to produce its lower limit, i.e., $p_{i}^{\text {min }}, s_{i}=1$ implies the $i$ th generator decides to produce its upper limit, i.e., $p_{i}^{\max }$, and $s_{i}=0$ implies the $i$ th generator wants to continue negotiation by receiving new values of $\delta, \beta$, and $P_{d}$ from the aggregator.

Proposition 1. Let $\mathbf{p}^{(0)}$ be given by (15) and define a recursive equation $\mathbf{p}^{(k)}:=F\left(\mathbf{p}^{(k-1)}\right)$, where $F: \mathbb{R}^{N} \longrightarrow \mathbb{R}^{N}$ is defined by (18). If there exists $p \in \Delta \triangleq\left\{\mathbf{p} \in \mathbb{R}^{N}: p_{i}^{\min } \leq \mathbf{p}_{i} \leq p_{i}^{\max } ; i=\right.$ $1,2, \ldots, N\}$ such that $\sum_{i=1}^{N} p_{i}=P_{d}$, then this recursive equation with the initial condition $\mathbf{p}^{(0)}$ given in (15) converges.

Proof. Let the optimal value of $\mathrm{ED}^{\prime}(\mathbf{p})$ in (17) be $J(\mathbf{p})$. Starting from the initial condition $\mathbf{p}^{(0)}$, we define a sequence of real numbers $\eta_{k}:=J\left(\mathbf{p}^{(k-1)}\right)$ for $k \geq 1$. From the update rule in (18), this sequence is monotonically nondecreasing, i.e., $\eta_{k} \leq$ $\eta_{k+1}$ for $k \geq 1$, and it has an upper bound since there exists $p \in \Delta \triangleq\left\{\mathbf{p} \in \mathbb{R}^{N}: p_{i}^{\min } \leq \mathbf{p}_{i} \leq p_{i}^{\max } ; i=1,2, \ldots, N\right\}$ such that $\sum_{i=1}^{N} p_{i}=P_{d}$. From the monotone convergence theorem [16], this sequence converges. Due to strict convexity of $f_{i}$ for all $i, \mathbf{p}^{(k)}$ is uniquely defined for each $\eta_{k}$ for all $k \geq 1$ and the convergence of $\eta_{k}$ implies the convergence of $\mathbf{p}^{(k)}$.

It is also not hard to see that any initial condition $\mathbf{p}^{(0)} \epsilon$ $\Delta$ guarantees convergence of $F: \mathbb{R}^{N} \longrightarrow \mathbb{R}^{N}$ defined by (18) to a unique solution of the economic dispatch (12). That is because $\mathscr{I}\left(\mathbf{p}^{(0)}\right)=\{1,2, \ldots, N\}$ and $\mathbf{p}^{(1)}$ is given as (15). Figure 3 shows the iterative message-passing between the aggregator (Node 0) and distributed generators (Nodes $1,2, \ldots, N)$. Notice that the broadcasting message announced by the aggregator at time step $k$ is a tuple $\left(\delta^{(k)}, \beta^{(k)}, P_{d}^{(k)}\right)$ that is computed from the collected private messages $\left\{m_{i}^{(k)}=\right.$ $\left.\left(a_{i}^{(k)}, b_{i}^{(k)}, s_{i}^{(k)}\right): i=1,2, \ldots, N\right\}$ by following (18).

Remark 2. In our iterative message-passing framework for distributed economic dispatch that is depicted in Figure 3, it is assumed that all generators report their true cost (function) 


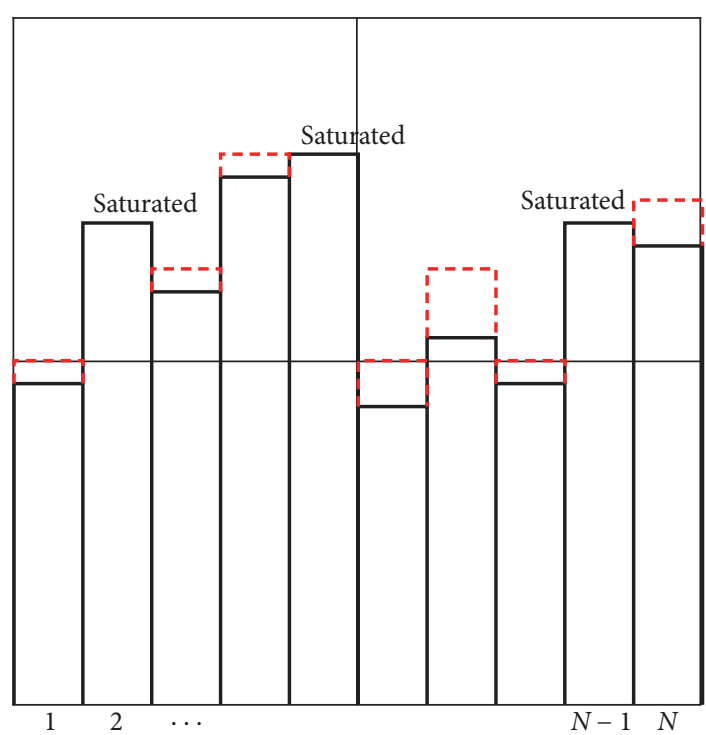

FIGURE 4: A schematic diagram of water-filling in decentralized multiagent learning. When power demand increases as $P_{d}^{\prime}$, each unsaturated generator $j$ that does not meet its capacity limit is required to produce additional power $\left(P_{d}^{\prime}-P_{d}\right)\left(\left(1 / a_{j}\right) / \sum_{i \in \widetilde{F}} 1 / a_{i}\right)$, where $\widetilde{\mathscr{I}}$ refers to the set of unsaturated generators.

parameters $\left(a_{i}, b_{i}\right), i=1,2, \ldots, N$. It is, of course, possible that a generator can take advantage of lying to the cost parameters. It is our future work to design a pricing mechanism computing $\mu$ based on the reported cost parameters so that none of generators can benefit from lying. Such a problem is related to the problem of parameterized supply function equilibrium [17-19].

In the presence of nontrivial transmission loss coefficients $B_{i j}$, the proposed linear iterative projection method has to be modified. A heuristic method can be applied to take the loss coefficients into account. At each iteration step $k$, the power demand $P_{d}^{(k)}$ is replaced by $P_{d}^{(k)}+\sum_{i=1}^{N} \sum_{j=1}^{N} p_{i}^{(k-1)} B_{i j} p_{j}^{(k-1)}$ that is computed and broadcasted by the aggregator as depicted in Figure 3. Upon a new residual power demand $P_{d}^{(k)}+$ $\sum_{i=1}^{N} \sum_{j=1}^{N} p_{i}^{(k-1)} B_{i j} p_{j}^{(k-1)}$ is computed, saturated generators are excluded in planning and allocation is performed among generators not yet reached the limits (see Figure 4).

\section{Further Characteristics of Economic Dispatch}

3.1. Convex Relaxation of QCQP. Consider the following optimization problem:

$$
\begin{array}{ll}
\operatorname{minimize} & \sum_{i=1}^{N} f_{i}\left(p_{i}\right) \\
\text { subject to } & p_{i}^{\min } \leq p_{i} \leq p_{i}^{\max } ; \quad i=1,2, \ldots, N \\
& \sum_{i=1}^{N} p_{i}-\sum_{i=1}^{N} \sum_{j=1}^{N} p_{i} B_{i j} p_{j} \geq P_{d}
\end{array}
$$

in which the equality constraint corresponding to demandsupply balance of ED in (1) is relaxed to be inequality. This can be rewritten as the following quadratically constrained quadratic programming (QCQP):

$$
\begin{array}{ll}
\operatorname{minimize} & \frac{1}{2} \mathbf{p}^{\top} A \mathbf{p}+b^{\top} \mathbf{p}+c \\
\text { subject to } & p^{\min } \leq \mathbf{p} \leq p^{\max } \\
& \mathbf{p}^{\top} B \mathbf{p}-1_{N}^{\top} \mathbf{p}+P_{d} \leq 0
\end{array}
$$

where $B$ is symmetric, but not necessarily positive semidefinite, so that this QCQP might be nonconvex. A method of convex relaxation can be applied to obtain the following semidefinite program (SDP):

$$
\begin{array}{cl}
\operatorname{minimize} & \frac{1}{2} \operatorname{tr}(A \mathbf{P})+b^{\top} \mathbf{p}+c \\
\text { subject to } & p^{\min } \leq \mathbf{p} \leq p^{\max } \\
& \operatorname{tr}(B \mathbf{P})-1_{N}^{\top} \mathbf{p}+P_{d} \leq 0 \\
& {\left[\begin{array}{cc}
\mathbf{P} & \mathbf{p} \\
\mathbf{p}^{\top} & 1
\end{array}\right] \succeq 0}
\end{array}
$$

where the linear matrix inequality is obtained from the Schur complement lemma for $\mathbf{P}-\mathbf{p p}^{\top} \geq 0$ This inequality corresponds to convexification of the equality $\mathbf{P}=\mathbf{p p}^{\top}$.

Lemma 3 (lossless relaxation). The optimal values and the associated solutions of (22) and (23) are the same.

Proof. Let a solution of the optimization (22) be $\overline{\mathbf{p}}$. Substituting $P=\overline{\mathbf{p}} \overline{\mathbf{p}}^{\top}+Q$ with $Q \geq 0$ into the optimization (23), we have

$$
\begin{array}{cl}
\text { minimize } & \frac{1}{2} \operatorname{tr}(A Q)+\frac{1}{2} \overline{\mathbf{p}}^{\top} A \overline{\mathbf{p}}+b^{\top} \overline{\mathbf{p}}+c \\
\text { subject to } & \operatorname{tr}(B Q)+\overline{\mathbf{p}}^{\top} B \overline{\mathbf{p}}-1_{N}^{\top} \overline{\mathbf{p}}+P_{d} \leq 0 \\
& Q \geq 0
\end{array}
$$

which reduces to

$$
\begin{array}{cl}
\text { minimize } & \operatorname{tr}(A Q) \\
\text { subject to } & \operatorname{tr}(B Q) \leq 0 \\
& Q \succeq 0
\end{array}
$$

where the constant factor $1 / 2$ in the objective function is removed for notational convenience. By considering a Lagrange function $L(Q, \lambda)=\operatorname{tr}((A+\lambda B) Q)$ and from the positive definiteness of $A>0$, the weak duality gives

$$
\text { optimal value of } \begin{aligned}
(25) & \geq \max _{\lambda \geq 0} \min _{Q \geq 0} L(Q, \lambda) \\
& = \begin{cases}0 & \text { if } A+\lambda B \geq 0 \\
-\infty & \text { otherwise }\end{cases}
\end{aligned}
$$


and this implies that the unique optimal solution of (25) is $Q^{*} \equiv 0$. Therefore, we conclude that the optimal solution of (23) is indeed the rank-one matrix $P^{*} \equiv \overline{\mathbf{p}} \overline{\mathbf{p}}^{\top}$ with an optimal solution $\overline{\mathbf{p}}$ of (22).

3.2. Multiparametric Programming. Consider the economic dispatch problem in (12). We analyze the dependence of the optimal solutions to the total power demand:

$$
p_{i}^{*}\left(P_{d}\right) \quad \text { for } i=1,2, \ldots, N
$$

Proposition 4. Suppose that there exists $p \in \Delta$ such that $\sum_{i=1}^{N} p_{i}=P_{d}$. Every $p_{i}^{*}\left(P_{d}\right)$ is monotonically nondecreasing and continuously piecewise affine in $P_{d} \geq 0$ for $i=1,2, \ldots, N$.

Proof. For the given $P_{d}>0$ and the corresponding optimal solution $p^{*}\left(P_{d}\right)$ of the optimization (12), consider the optimization (17) with $\mathbf{p}=p^{*}\left(P_{d}\right)$ and the associated computation (18). Then, there exists a small enough $\epsilon>0$ such that

$$
\begin{aligned}
& p_{i}^{*}\left(P_{d}+\epsilon\right) \\
& = \begin{cases}p_{i}^{\min } & \text { for } i \in \mathscr{I}_{\min }\left(\mathbf{p}^{*}\left(P_{d}\right)\right) \\
p_{i}^{\max } & \text { for } i \in \mathscr{I}_{\max }\left(\mathbf{p}^{*}\left(P_{d}\right)\right) \\
p_{i}^{*}\left(P_{d}\right)+\frac{1 / a_{i}}{\sum_{j \in \mathscr{I}} 1 / a_{j}} \epsilon & \text { for } i \in \widetilde{\mathscr{I}}\left(\mathbf{p}^{*}\left(P_{d}\right)\right)\end{cases}
\end{aligned}
$$

is the optimal solution of the economic dispatch (12) when the power demand is given by $P_{d}+\epsilon$. In other words, the index sets $\mathscr{I}_{\min }\left(\mathbf{p}^{*}\left(P_{d}+\epsilon\right)\right)$ and $\mathscr{I}_{\max }\left(\mathbf{p}^{*}\left(P_{d}\right)\right)$ remain the same and the optimal solutions $p_{i}^{*}\left(P_{d}\right)$ corresponding to the index set $\widetilde{\mathscr{I}}\left(\mathbf{p}^{*}\left(P_{d}\right)\right)$ change as $p_{i}^{*}\left(P_{d}\right)+\left(\left(1 / a_{i}\right) / \sum_{j \in \widetilde{\mathscr{I}}} 1 / a_{j}\right) \epsilon$. This shows that optimal solution $p^{*}\left(P_{d}\right)$ is (elementwise) monotonically nondecreasing and continuously piecewise affine in $P_{d} \geq 0$.

Now we analyze the explicit dependence of the optimal power generation profile $\mathbf{p}^{*}$ on the power demand $P_{d}$ for which the results from multiparametric programming (MPP) [20] are exploited. We can rewrite the problem of economic dispatch in (12) as the following quadratic programming:

$$
\begin{aligned}
V^{*}\left(P_{d}\right):= & \text { minimize } \\
\text { subject to } & \frac{1}{2} \mathbf{p}^{\top} A \mathbf{p}+b^{\top} \mathbf{p}+c \\
& H r+E P_{d}
\end{aligned}
$$

where

$$
A=\left[\begin{array}{cccc}
a_{1} & 0 & \cdots & 0 \\
0 & a_{2} & \ddots & \vdots \\
\vdots & \ddots & \ddots & 0 \\
0 & \cdots & 0 & a_{N}
\end{array}\right]
$$

$$
\begin{aligned}
& b=\left[\begin{array}{c}
b_{1} \\
b_{2} \\
\vdots \\
b_{N}
\end{array}\right], \\
& c=\sum_{i=1}^{N} c_{i}
\end{aligned}
$$$$
H=\left[\begin{array}{c}
1_{N \times 1}^{\top} \\
-1_{N \times 1}^{\top} \\
I_{N \times N} \\
I_{N \times N}
\end{array}\right],
$$$$
r=\left[\begin{array}{c}
0 \\
0 \\
\mathbf{p}^{\max } \\
-\mathbf{p}^{\min }
\end{array}\right],
$$

$$
E=\left[\begin{array}{c}
1 \\
1 \\
0_{N \times 1} \\
0_{N \times 1}
\end{array}\right]
$$

with $1_{N \times 1}$ all ones vector in $\mathbb{R}^{N}, 0_{N \times 1}$ all zeros vector in $\mathbb{R}^{N}, I_{N \times N}$ identity matrix in $\mathbb{R}^{N \times N}$, and $\mathbf{p}^{\max (\min )}=$ $\left[p_{1}^{\max (\min )} p_{2}^{\max (\min )} \cdots p_{N}^{\max (\min )}\right]^{\top} \in \mathbb{R}^{N}$. The associated KKT conditions are given by

$$
\begin{aligned}
A \mathbf{p}+H^{\top} \mathbf{w}=0 & (\text { stationarity }) \\
H \mathbf{p}-r-E P_{d} \leq 0 & (\text { primal feasibility }) \\
\mathbf{w} \geq 0 & (\text { dual feasibility }) \\
\mathbf{w}^{\top}\left(H \mathbf{p}-r-E P_{d}\right)=0 & (\text { complementarity })
\end{aligned}
$$

where $\mathbf{w} \in \mathbb{R}^{(2 N+2)}$ is a (vector) Lagrange multiplier corresponding to the inequality in (29). For given $P_{d}$ with which the optimization in (29) can be solved, the corresponding optimizer $\mathbf{p}$ yields the set of active constraints defined as

$$
\mathscr{I}_{a}\left(P_{d}\right) \triangleq\left\{i \in \mathscr{J}: H_{i} \mathbf{p}=r_{i}+E_{i} P_{d}\right\}
$$

where $\mathscr{J}=\{1,2, \ldots, 2 N+2\}$ and $(\cdot)_{i}$ denotes the $i$ th row of the argument. The rows of the constraints matrices $H, r$, and $E$ corresponding to the index set $\mathscr{I}_{a}\left(P_{d}\right)$ are extracted to construct the matrices $H_{\mathscr{I}_{a}\left(P_{d}\right)}, r_{\mathscr{I}_{a}\left(P_{d}\right)}$, and $E_{\mathscr{I}_{a}\left(P_{d}\right)}$ that would be rewritten in the following compact forms: $H_{\mathscr{I}_{a}}$, $r_{\mathscr{I}_{a}}$, and $E_{\mathscr{I}_{a}}$, respectively, by hiding its dependence of $P_{d}^{a}$. By definition of $\mathscr{I}_{a}\left(P_{d}\right)$, we have the equality conditions of optimality

$$
H_{\mathscr{I}_{a}} \mathbf{p}-r_{\mathscr{J}_{a}}-E_{\mathscr{I}_{a}} P_{d}=0
$$


Combining the two systems of equations (31) and (36) gives

$$
-H_{\mathscr{J}_{a}} A^{-1} H^{\top} \mathbf{w}-r_{\mathscr{I}_{a}}-E_{\mathscr{I}_{a}} P_{d}=0
$$

where $H^{\top} \mathbf{w}=H_{\mathscr{F}_{a}}^{\top} \mathbf{w}_{\mathscr{I}_{a}}$ because the complementarity condition (34) implies $\mathbf{w}_{\mathscr{F}_{a}^{c}}=0$ for $\mathscr{I}_{a}^{\mathrm{c}}=\mathscr{J} \backslash \mathscr{I}_{a}$. Therefore, we obtain an active (vector) Lagrange multiplier

$$
\begin{aligned}
\mathbf{w}= & -\left(H_{\mathscr{I}_{a}} A^{-1} H_{\mathscr{J}_{a}}^{\top}\right)^{-1} r_{\mathscr{I}_{a}} \\
& -\left(H_{\mathscr{I}_{a}} A^{-1} H_{\mathscr{I}_{a}}^{\top}\right)^{-1} E_{\mathscr{I}_{a}} P_{d}
\end{aligned}
$$

and the corresponding (primal) optimizer

$$
\begin{aligned}
\mathbf{p}= & -A^{-1} H_{\mathscr{I}_{a}}^{\top} \mathbf{w}_{\mathscr{I}_{a}} \\
= & A^{-1} H_{\mathscr{I}_{a}}^{\top}\left(H_{\mathscr{J}_{a}} A^{-1} H_{\mathscr{I}_{a}}^{\top}\right)^{-1} E_{\mathscr{I}_{a}} P_{d} \\
& +A^{-1} H_{\mathscr{I}_{a}}^{\top}\left(H_{\mathscr{I}_{a}} A^{-1} H_{\mathscr{I}_{a}}^{\top}\right)^{-1} r_{\mathscr{J}_{a}}
\end{aligned}
$$

where the inverse exists provided that the active constraints are linearly independent. Note that the active Lagrange multiplier and optimizer obtained in (38) and (39), respectively, are affine functions in $P_{d}$. The next step is to find or characterize all the points $P_{d}^{\prime}$ in the neighborhood of $P_{d}$ in which the optimizer $\mathbf{p}\left(P_{d}^{\prime}\right)$ has the same set of active constraints, i.e., $\mathscr{I}_{a}\left(P_{d}^{\prime}\right)=\mathscr{I}_{a}\left(P_{d}\right)$ so that $\mathbf{p}\left(P_{d}^{\prime}\right)$ is of the same form as (39). Such neighborhood of $P_{d}$ can be obtained by substituting (39) and (38) into (32) and (33), respectively. Furthermore, it turns out that the neighborhood is a polyhedron

$$
\mathscr{P}_{\ell} \triangleq\left\{P_{d}^{\prime} \in \mathbb{R}_{+}: Q_{\ell} P_{d}^{\prime} \leq q_{\ell}\right\}
$$

where

$$
\begin{aligned}
Q_{\ell} & =\left[\begin{array}{c}
H A^{-1} H_{\mathscr{F}_{a}}^{\top}\left(H_{\mathscr{I}_{a}} A^{-1} H_{\mathscr{F}_{a}}^{\top}\right)^{-1} E_{\mathscr{I}_{a}}-E \\
\left(H_{\mathscr{J}_{a}} A^{-1} H_{\mathscr{I}_{a}}^{\top}\right)^{-1} E_{\mathscr{I}_{a}}
\end{array}\right], \\
q_{\ell} & =\left[\begin{array}{c}
r-H A^{-1} H_{\mathscr{I}_{a}}^{\top}\left(H_{\mathscr{I}_{a}} A^{-1} H_{\mathscr{I}_{a}}^{\top}\right)^{-1} r_{\mathscr{I}_{a}} \\
-\left(H_{\mathscr{I}_{a}} A^{-1} H_{\mathscr{I}_{a}}^{\top}\right)^{-1} r_{\mathscr{I}_{a}}
\end{array}\right]
\end{aligned}
$$

are computed from a given $P_{d}$ and the associated optimizer $\mathbf{p}\left(P_{d}\right)$.

Remark 5. We have shown that the affine dependence of the optimal power generation profiles on the power demand can be explicitly analyzed. This parameterization could be useful when the power demands are not known a priori but forecasted with uncertainty. Analyzing the required power generation profiles over the wide range of power demands offline would help the system operator to schedule the power supply in smaller time-scales.

3.3. Uncertain Transmission Loss and Power Demand. In Section 3.2, a method of multiparametric programming is suggested for taking care of uncertain power demand $P_{d}$. By applying MPP, economic generation dispatch is computed as a function of $P_{d}$ in (38) and (39). In addition to uncertain power demand $P_{d}$, we also need to consider uncertain or variable power loss $P_{L}$ that is parameterized as a quadratic function $\sum_{i=1}^{N} \sum_{j=1}^{N} p_{i} B_{i j} p_{j}$. The loss coefficients $B_{i j}$ are not certain but can be assumed to be bounded as

$$
B_{i j}^{\min } \leq B_{i j} \leq B_{i j}^{\max }, \quad \text { for } i, j=1,2, \ldots, N
$$

where $B_{i j}^{\min }$ and $B_{i j}^{\max }$ refer to the lower and upper bounds of $B_{i j}$, respectively. For more general representation of uncertain loss coefficient matrix $B$, we assume that $B$ is not fixed but belongs to a convex polytope that has a finite number of vertices:

$$
B \in \operatorname{Conv}\left\{B^{(0)}, B^{(1)}, \ldots, B^{(\ell)}\right\}
$$

where $\operatorname{Conv}\{\cdot\}$ denotes the convex hull of a set. In other words, there exists $t_{0}, t_{1}, \ldots, t_{\ell} \in[0,1]$ with $\sum_{\tau=0}^{\ell} t_{\tau}=1$ such that $B=\sum_{\tau=0}^{\ell} t_{\tau} B^{(\tau)}$. This implies that the quadratic loss function is parameterized as $P_{L}(p)=\sum_{\tau=0}^{\ell} \sum_{i=1}^{N} \sum_{j=1}^{N} t_{k} p_{i} B_{i j}^{(\tau)} p_{j}$, where $t_{\tau} \in[0,1]$ are not known but uncertain. To take care of uncertain loss coefficients, the three proposed methods need to be modified.

Modified SDP Method. The SDP method presented in (23) is modified to take the uncertain loss coefficient matrix $B$ in (43) as follows:

$$
\begin{array}{ll}
\text { minimize } & \frac{1}{2} \operatorname{tr}(A \mathbf{P})+b^{\top} \mathbf{p}+c \\
\text { subject to } & p^{\min } \leq \mathbf{p} \leq p^{\max } \\
& \operatorname{tr}\left(B^{(\tau)} \mathbf{P}\right)-1_{N}^{\top} \mathbf{p}+P_{d} \leq 0, \\
& \quad \text { for } \tau=0,1, \ldots, \ell \\
& {\left[\begin{array}{cc}
\mathbf{P} & \mathbf{p} \\
\mathbf{p}^{\top} & 1
\end{array}\right] \succeq 0}
\end{array}
$$

that is indeed robust optimization counterpart of (23).

Modified Iterative Primal-Dual Method. The iterative primaldual method presented in (7) and (11) is modified to take the uncertain loss coefficient matrix $B$ in (43) as follows:

$$
\begin{aligned}
& p_{i}^{(k+1)}:=\mathrm{BR}_{i}\left(p_{\mathcal{N}_{i}}^{(k)}, \mu^{(k)}\right) \quad \text { for } i=1,2, \ldots, N \\
& \mu^{(k+1)}:=\mu^{(k)}+\alpha^{(k+1)}\left(P_{d}+P_{L}^{(k+1)}-\sum_{i=1}^{N} p_{i}^{(k+1)}\right)
\end{aligned}
$$

where

$$
P_{L}^{(k+1)}:=\max _{0 \leq \tau \leq \ell}\left\{\sum_{i=1}^{N} \sum_{j \in \mathcal{N}_{i}} p_{i}^{(k+1)} B_{i j}^{(\tau)} p_{j}^{(k+1)}\right\}
$$

that is the worst-case line loss for given $p_{i}^{(k+1)}, i=1,2, \ldots, N$. 
TABLE 1: Problem data for a test case of 6 generators.

\begin{tabular}{lccccc}
\hline $\begin{array}{l}\text { Generator } \\
i\end{array}$ & $\begin{array}{c}a_{i} \\
{\left[\$ / \mathrm{MW}^{2}\right]}\end{array}$ & $\begin{array}{c}b_{i} \\
{[\$ / \mathrm{MW}]}\end{array}$ & $\begin{array}{c}c_{i} \\
{[\$]}\end{array}$ & $\begin{array}{c}p_{i}^{\min } \\
{[\mathrm{MW}]}\end{array}$ & $\begin{array}{c}p_{i}^{\max } \\
{[\mathrm{MW}]}\end{array}$ \\
\hline 1 & 0.00375 & 2.00 & 0 & 20 & 20 \\
2 & 0.01750 & 1.75 & 0 & 15 & 80 \\
3 & 0.06250 & 1.00 & 0 & 10 & 30 \\
4 & 0.00834 & 3.25 & 0 & 10 & 35 \\
5 & 0.02500 & 3.00 & 0 & 12 & 40 \\
6 & 0.02500 & 3.00 & 0 & \\
\hline
\end{tabular}

Modified Iterative Linear Projection Method. The iterative primal-dual method presented in (19) is modified to take the uncertain loss coefficient matrix $B$ in (43) as follows:

$$
p_{i}^{(k+1)}= \begin{cases}p_{i}^{\min } & \text { for } i \in \mathscr{I}_{\min }\left(\mathbf{p}^{(k)}\right) \\ p_{i}^{\max } & \text { for } i \in \mathscr{I}_{\max }\left(\mathbf{p}^{(k)}\right) \\ \frac{1}{a_{i}}\left(\widetilde{\delta} \widetilde{\beta}+\widetilde{\delta} \widetilde{P}_{d}-b_{i}\right) & \text { for } i \in \widetilde{\mathscr{I}}\left(\mathbf{p}^{(k)}\right)\end{cases}
$$

where

$$
\begin{aligned}
\widetilde{P}_{d}= & P_{d}+\max _{0 \leq \tau \leq \ell}\left\{\sum_{i=1}^{N} \sum_{j \in \mathcal{N}_{i}} p_{i}^{(k)} B_{i j}^{(\tau)} p_{j}^{(k)}\right\} \\
& -\sum_{i \in \mathcal{F}_{\max }\left(\mathbf{p}^{(k)}\right)} p_{i}^{\max }-\sum_{i \in \mathcal{F}_{\min }\left(\mathbf{p}^{(k)}\right)} p_{i}^{\min },
\end{aligned}
$$

and $\widetilde{\delta}$ and $\widetilde{\beta}$ are defined the same as (19).

\section{Case Study}

To demonstrate our methods of economic dispatch presented in Sections 2 and 3, we consider the 6-generator test case whose generation cost parameters and generation limits are given in Table 1 . This system contains six generation units, 26 buses, and 46 transmission lines [10]. The power loss function $\mathbf{p}^{\top} B \mathbf{p}$ is parameterized with a symmetric matrix of loss coefficients that is given by

$B$

$$
=10^{-3}\left[\begin{array}{cccccc}
0.218 & 0.103 & 0.009 & -0.010 & 0.002 & 0.027 \\
0.103 & 0.181 & 0.004 & -0.015 & 0.002 & 0.030 \\
0.009 & 0.004 & 0.417 & -0.131 & -0.153 & -0.107 \\
-0.010 & -0.015 & -0.131 & 0.221 & 0.094 & 0.050 \\
0.002 & 0.002 & -0.153 & 0.094 & 0.243 & -0.000 \\
0.027 & 0.030 & -0.107 & 0.050 & -0.000 & 0.358
\end{array}\right]
$$

Three different methods, (a) SDP method (centralized), (b) iterative primal-dual method (distributed), and (c) iterative linear projection method (distributed), are applied to find optimal solutions of economic dispatch with varying power demands. All numerical computations are carried out using MATLAB on a $1.6 \mathrm{GHz}$ Intel Core i5 personal laptop with $8 \mathrm{~GB}$ $1600 \mathrm{MHz}$ DDR3.

Optimality. The resultant optimal power generation profiles computed by the proposed three optimization algorithms are presented in Figure 5. For power demands varying from 117 [MW] to 435 [MW], we observe that the three different methods give the exactly same results of economic generation dispatch. Generators 1 and 2 have smaller $a_{i}$ with larger upper bounds $p_{i}^{\max }$, so that they become active in the sense that $p_{i}^{*}>$ $p_{i}^{\text {min }}$ when $P_{d}>P_{d}^{\min }$. When Generator 1 with the smallest marginal cost is saturated, i.e., $p_{1}^{*}=p_{1}^{\max }$, as $P_{d}$ increases, the slopes corresponding to change rates of Generators 2 and 3 become larger. This is because the value of $\delta=$ $\left(\sum_{i=1}^{N} 1 / a_{i}\right)^{-1}$ changes to the larger value $\left(\sum_{i \neq 1}^{N} 1 / a_{i}\right)^{-1}$. Such changes in slopes occur whenever a generator is saturated in its upper limit. As a matter of fact, such changes in activeness and slopes are explicitly exploited in the iterative linear projection method that significantly reduces computational complexity.

Computational Complexity. In Figure 6, we compare the computation times of the three proposed methods. It shows the histograms of the three different methods to compute the optimal economic dispatch profiles with varying total power demand ranging from 117 [MW] to 435 [MW]. Since the computation times heavily depend on the choice of the initial guesses of the primal and dual variables and the algorithms for solving semidefinite programs, we do not exploit any warm-start strategies for fair comparisons. We observe that the algorithm of iterative linear projection requires a significantly small size of iterations and tremendously fast speed in computations - it is three orders of magnitude faster than the primal-dual iteration method in average. The distributed algorithm based on primal-dual iteration is approximately three times faster than the centralized semidefinite program in average. The SDP method presented in (23) requires a test for positive semidefiniteness of a symmetric matrix whose size is $N+1$ with $N(N+1) / 2+N$ variables at each NewtonKKT iteration step of the interior-point method [21]. Such test has computational complexity $O\left(\left(\left(N^{2}+3 N\right) / 2\right)(N+\right.$ 


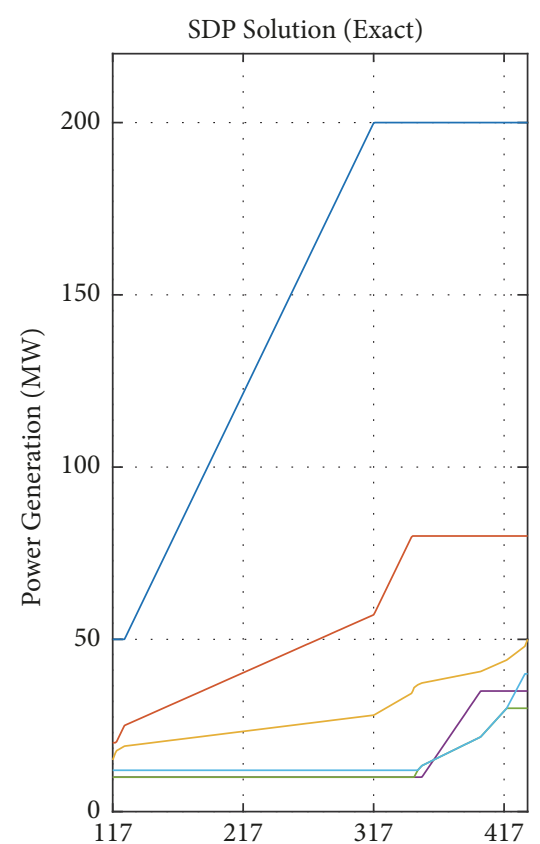

Power Generation Profiles

- Generator 1

- Generator 2

- Generator 3

- Generator 4

- Generator 5

- Generator 6

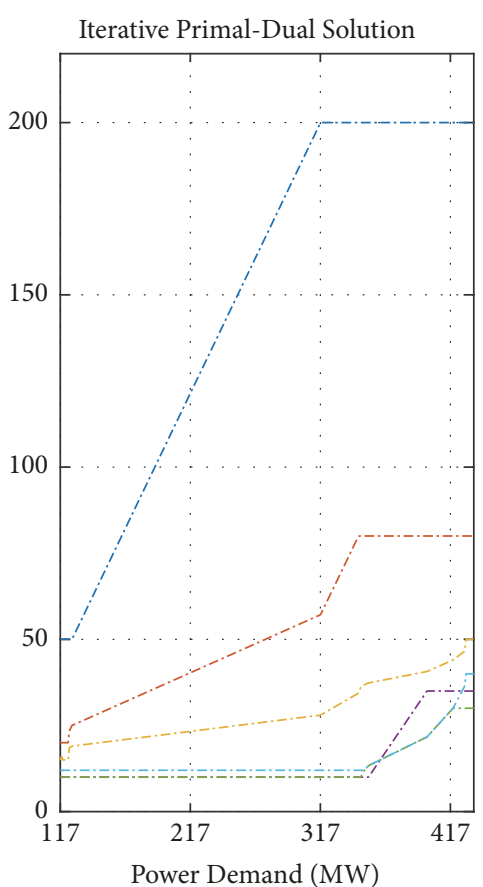

Power Generation Profiles

-.... Generator 1

-.... Generator 2

-.... Generator 3

-.... Generator 4

-... Generator 5

-... Generator 6

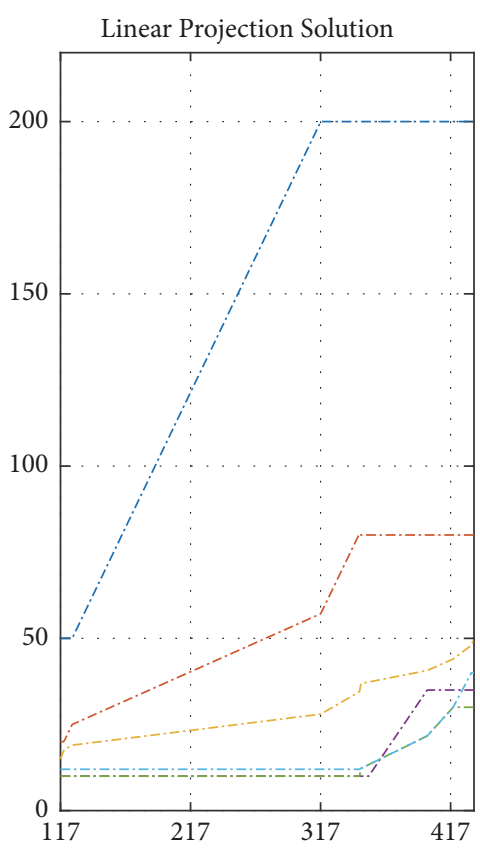

Power Generation Profiles

-.... Generator 1

-... Generator 2

-.... Generator 3

-... Generator 4

-.... Generator 5

-... Generator 6

FIGURE 5: Economic dispatch solutions for varying power demands (from 117 [MW] to 435 [MW]) obtained by three different methods: (a) SDP method, (b) iterative primal-dual method, and (c) iterative linear projection method.

$\left.1)^{4}\right)+(N+1)^{\mathrm{O}\left(\min \left\{(N+1)^{2},\left(N^{2}+3 N\right) / 2\right\}\right)}$ in terms of floating-point operations [22], without exploiting any sparsity structures in problem. The primal-dual iteration method presented in (7) and (11) requires iterative projections that are decomposed into one-dimensional subspaces in primal updates and linear recursive computations in dual updates. Such procedure has computational complexity $O\left(N^{2}\right)$. The convergence rate is heavily dependent on the step-size $\alpha^{(k)}$ and we choose $\alpha^{(k)}>$ 0 such that $\sum \alpha^{(k)}=\infty$ and $\sum\left(\alpha^{(k)}\right)^{2}<\infty$. The iterative linear projection method presented in (19) requires computations of multiplication and addition. Its computational complexity is $O(N)$ and more importantly, the projection terminates in less than $N$ steps. This reduces significant amounts of computation time.

\section{Conclusion}

In this paper, we present three different methods of solving problems of economic dispatch with transmission losses. The iterative primal-dual method is indeed based on indirect approach to optimization in which the KKT conditions are solved in a distributed manner. The iterative linear projection method is based on closed-form solutions for the optimality conditions of unconstrained counterparts and the closedform solutions are iteratively projected on the set corresponding to the capacity limits of generation units. The SDP method is based on the convex relaxation of the associated quadraticconstrained quadratic program whose exactness is shown from the strong duality. The parametric dependence of the power generation profiles is also analyzed with varying power demands in economic dispatch.

\section{Data Availability}

No data were used to support this study.

\section{Conflicts of Interest}

The author declares that they have no conflicts of interest regarding the publication of this paper.

\section{Acknowledgments}

This work was supported by Inha University Grant (INHA57823) and Korea Electric Power Corporation (Grant number: R18XA01). 
SDP Solution (Exact)

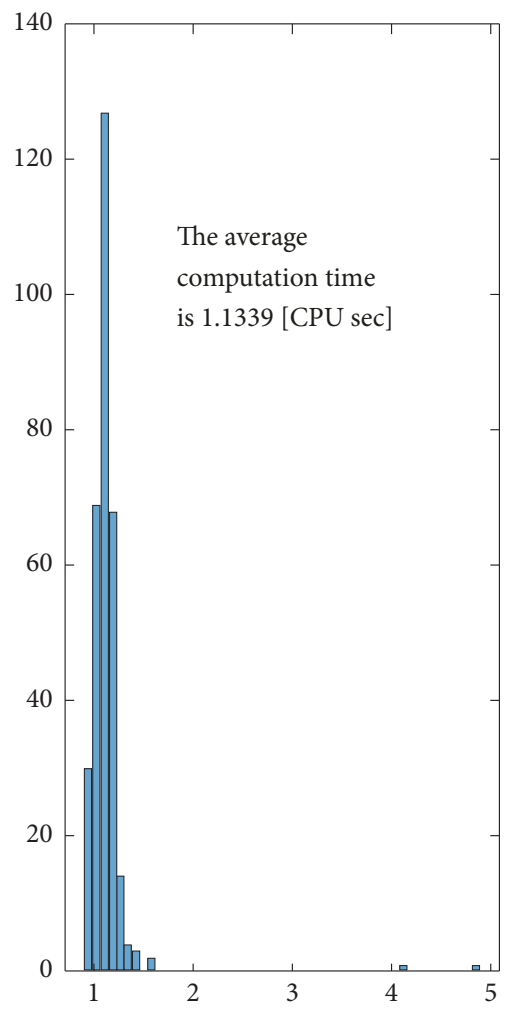

Iterative Primal-Dual Solution

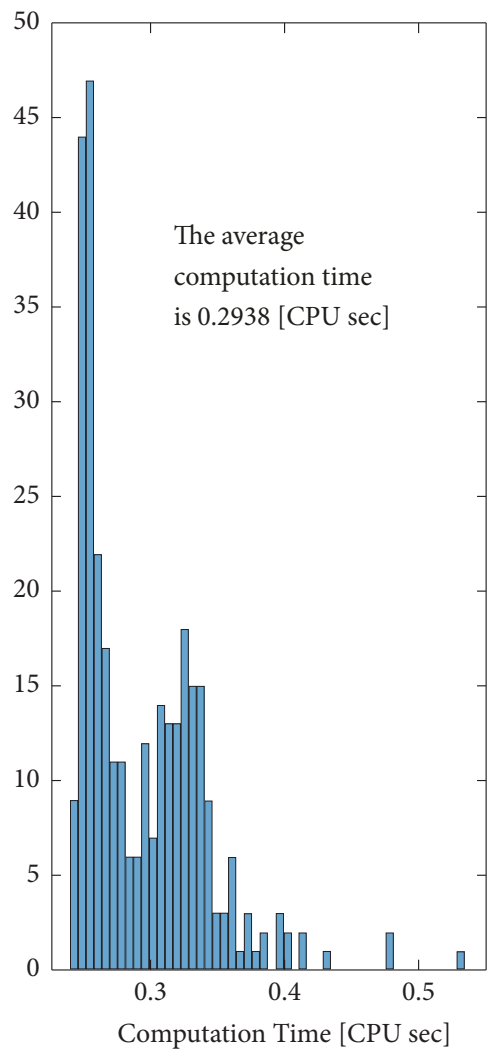

Linear Projection Solution

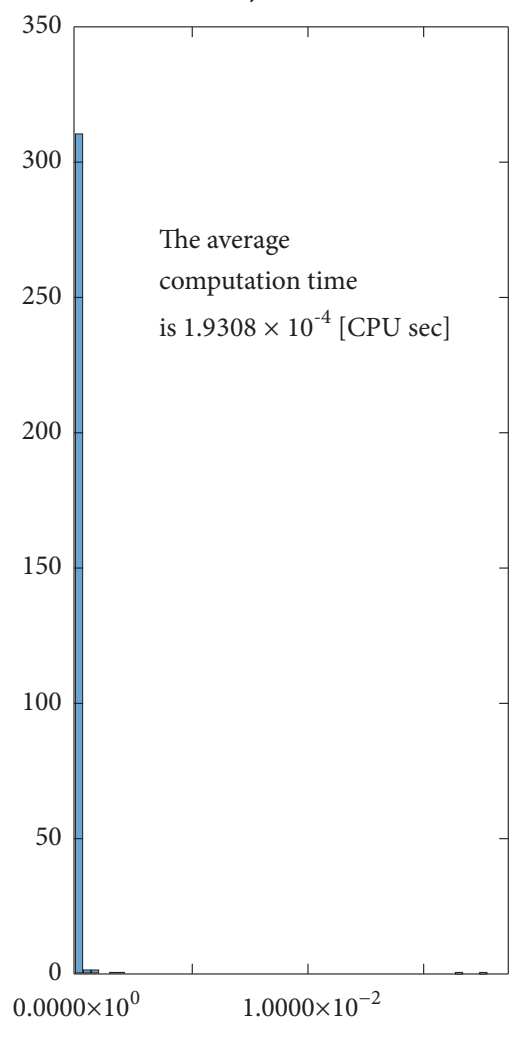

FIGURE 6: Comparison of computation times for three different numerical methods: (a) SDP method, (b) iterative primal-dual method, and (c) iterative linear projection method.

\section{References}

[1] K. Heussen, A. Saleem, and M. Lind, "Control architecture of power systems: Modeling of purpose and function," in Proceedings of the 2009 IEEE Power and Energy Society General Meeting, PES '09, 8, 1 pages, July 2009.

[2] D. K. Molzahn, F. Dörfler, H. Sandberg et al., "A survey of distributed optimization and control algorithms for electric power systems," IEEE Transactions on Smart Grid, vol. 8, no. 6, pp. 2941-2962, 2017.

[3] B. H. Chowdhury and S. Rahman, "A review of recent advances in economic dispatch," IEEE Transactions on Power Systems, vol. 5, no. 4, pp. 1248-1259, 1990.

[4] Z. Zhang, X. Ying, and M.-Y. Chow, "Decentralizing the economic dispatch problem using a two-level incremental cost consensus algorithm in a smart grid environment," in Proceedings of the IEEE North American Power Symposium (NAPS), pp. 1-7, 2011.

[5] Z. Zhang and M. Chow, "Convergence analysis of the incremental cost consensus algorithm under different communication network topologies in a smart grid," IEEE Transactions on Power Systems, vol. 27, no. 4, pp. 1761-1768, 2012.

[6] S. Yang, S. Tan, and J.-X. Xu, "Consensus based approach for economic dispatch problem in a smart grid," IEEE Transactions on Power Systems, vol. 28, no. 4, pp. 4416-4426, 2013.

[7] H. Xing, Y. Mou, M. Fu, and Z. Lin, "Consensus based bisection approach for economic power dispatch," in Proceedings of the
2014 53rd IEEE Annual Conference on Decision and Control, CDC 2014, pp. 3789-3794, December 2014.

[8] H. Xing, Y. Mou, M. Fu, and Z. Lin, "Distributed bisection method for economic power dispatch in smart grid," IEEE Transactions on Power Systems, vol. 30, no. 6, pp. 3024-3035, 2015.

[9] H. Xing, Z. Lin, and M. Fu, "Distributed augmented lambdaiteration method for economic dispatch in smart grid," in Proceedings of the 2017 Chinese Automation Congress, CAC 2017, pp. 3302-3307, China, October 2017.

[10] H. Saadat, Power System Analysis, McGraw-Hill Book Co., New York, NY, USA, 1999.

[11] A. Pantoja and N. Quijano, "A population dynamics approach for the dispatch of distributed generators," IEEE Transactions on Industrial Electronics, vol. 58, no. 10, pp. 4559-4567, 2011.

[12] P. Chakraborty, E. Baeyens, P. P. Khargonekar, and K. Poolla, "A cooperative game for the realized profit of an aggregation of renewable energy producers," in Proceedings of the 55th IEEE Conference on Decision and Control, CDC 2016, pp. 5805-5812, USA, December 2016.

[13] C. Chiang, "Improved genetic algorithm for power economic dispatch of units with valve-point effects and multiple fuels," IEEE Transactions on Power Systems, vol. 20, no. 4, pp. 16901699, 2005.

[14] Z. Gaing, "Particle swarm optimization to solving the economic dispatch considering the generator constraints," IEEE Transactions on Power Systems, vol. 18, no. 3, pp. 1187-1195, 2003. 
[15] N. Sinha, R. Chakrabarti, and P. K. Chattopadhyay, "Evolutionary programming techniques for economic load dispatch," IEEE Transactions on Evolutionary Computation, vol. 7, no. 1, pp. 8394, 2003.

[16] W. Rudin, Principles of Mathematical Analysis, McGraw-Hill Book Co., New York, NY, USA, 1976.

[17] R. Baldick, "Electricity market equilibrium models: the effect of parametrization," IEEE Transactions on Power Systems, vol. 17, no. 4, pp. 1170-1176, 2002.

[18] R. Johari and J. N. Tsitsiklis, "Parameterized supply function bidding: equilibrium and efficiency," Operations Research, vol. 59, no. 5, pp. 1079-1089, 2011.

[19] W. Lin and E. Bitar, "Parameterized supply function equilibrium in power networks," in Proceedings of the 55th IEEE Conference on Decision and Control, CDC 2016, pp. 1542-1548, December 2016.

[20] E. N. Pistikopoulos, N. A. Diangelakis, and R. Oberdieck, Multiparametric Optimization and Control, Wiley-VCH, New York, NY, USA, 2018.

[21] L. Vandenberghe and S. Boyd, "Semidefinite programming," SIAM Review, vol. 38, no. 1, pp. 49-95, 1996.

[22] L. Porkolab and L. Khachiyan, "On the complexity of semidefinite programs," Journal of Global Optimization, vol. 10, no. 4, pp. 351-365, 1997. 


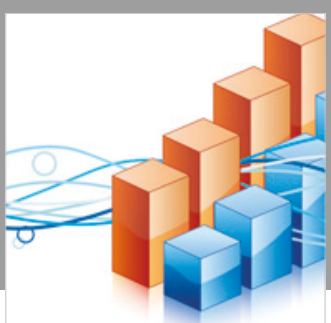

Advances in

Operations Research

\section{-n-m}
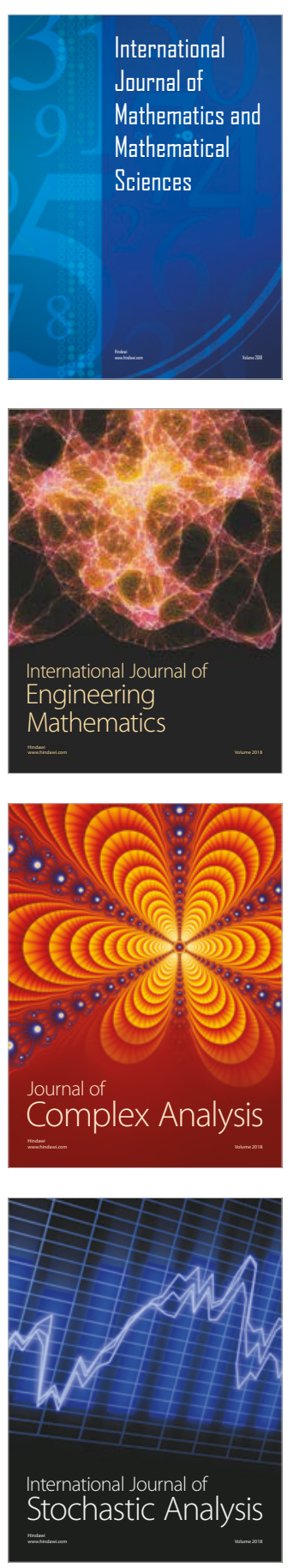
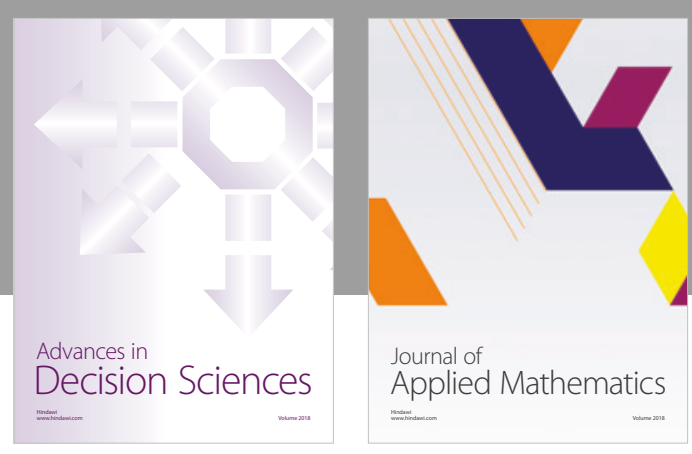

Journal of

Applied Mathematics
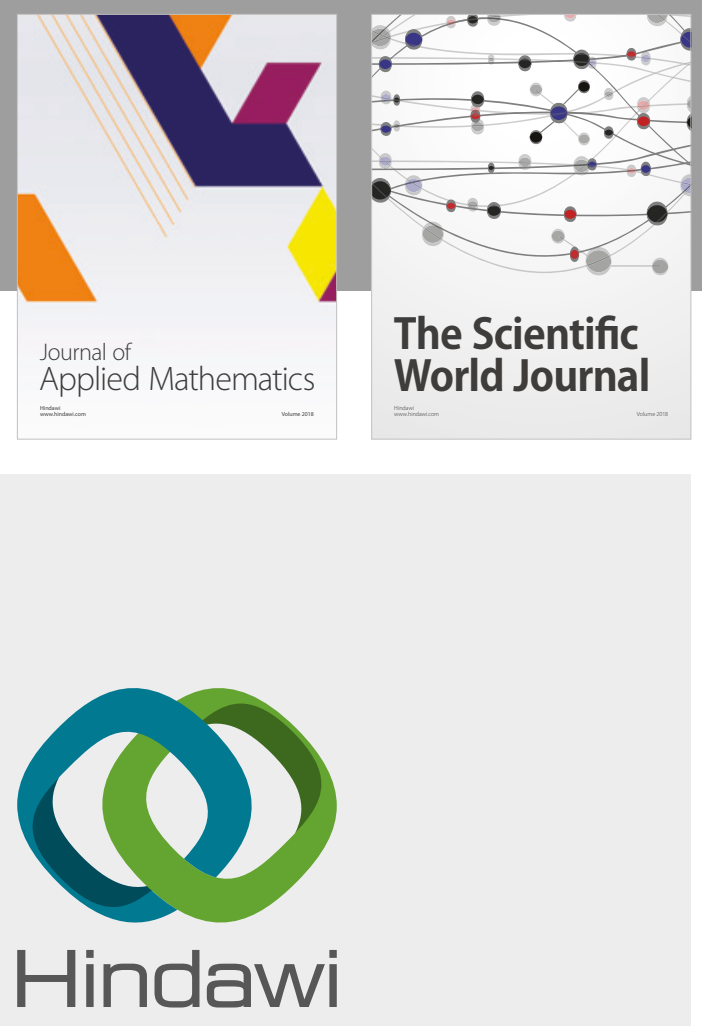

Submit your manuscripts at

www.hindawi.com

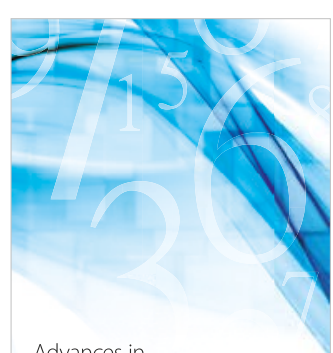

Advances in
Numerical Analysis
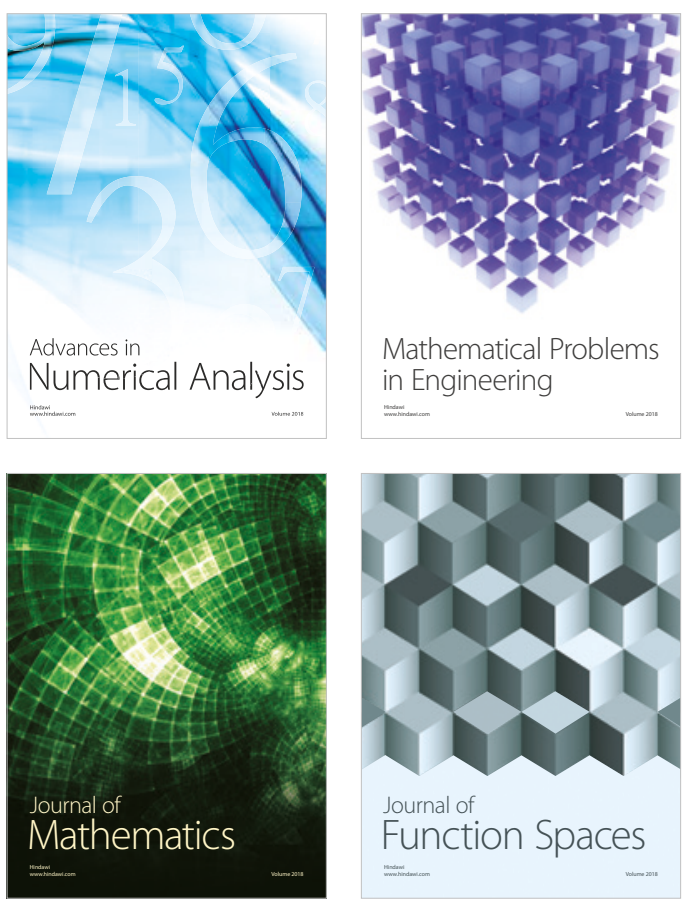

Mathematical Problems in Engineering

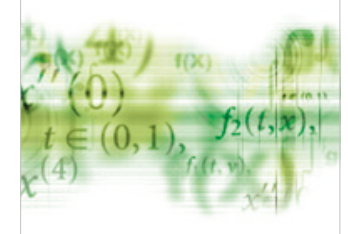

International Journal of

Differential Equations

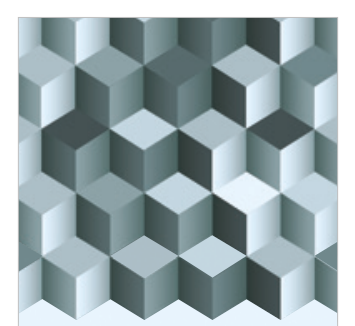

Journal of

Function Spaces

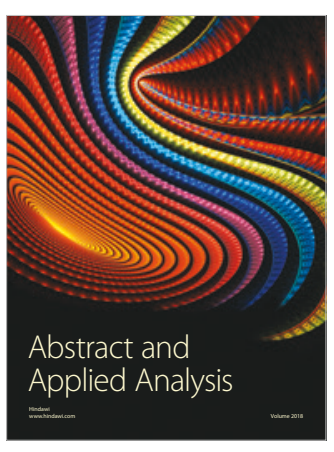

The Scientific

World Journal

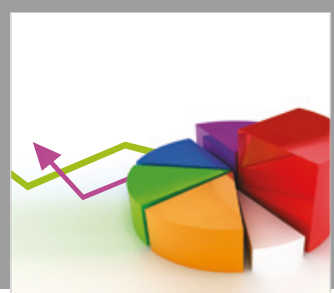

Journal of

Probability and Statistics
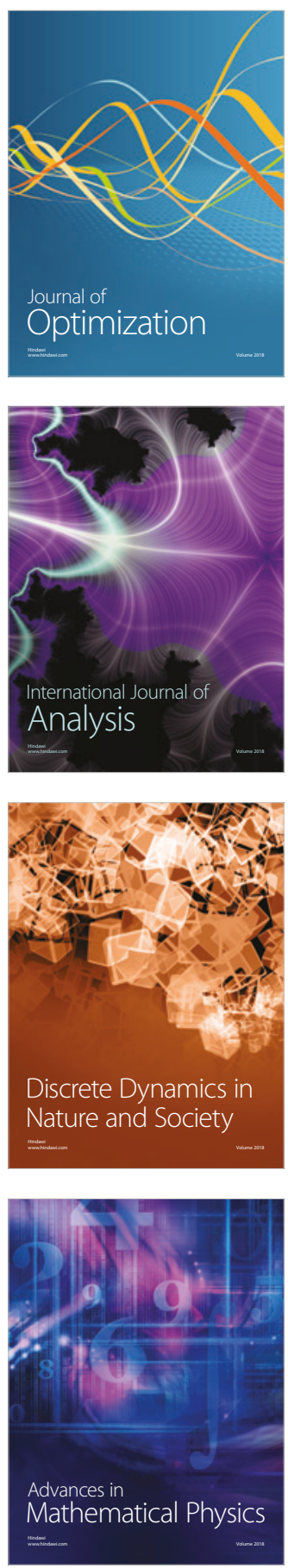